\title{
Do TV viewing and frequency of ultra-processed food consumption share mediators in relation to adolescent anxiety-induced sleep disturbance?
}

\author{
André $\bigcirc$ Werneck ${ }^{1, *}$ (1), Erin Hoare ${ }^{2}$ and Danilo R Silva ${ }^{3}$ \\ 'Department of Nutrition, School of Public Health, University of São Paulo (USP), Av. Dr. Arnaldo, 715 - Cerqueira \\ César, São Paulo, SP 01246-904, Brazil: ${ }^{2} F o o d \&$ Mood Centre, Centre for Innovation in Mental and Physical Health \\ and Clinical Treatment, School of Medicine, Faculty of Health, Deakin University, Melbourne, VIC, Australia: \\ ${ }^{3}$ Department of Physical Education, Federal University of Sergipe - UFS, São Cristóvão, Brazil
}

Submitted 7 July 2020: Final revision received 7 January 2021: Accepted 20 January 2021: First published online 27 January 2021

\begin{abstract}
Objective: To investigate the role of potential shared mediators in the association of TV viewing and frequency of ultra-processed food consumption with anxietyinduced sleep disturbance.

Design: Cross-sectional study.

Setting: Data from the Adolescent School-Based Health Survey, a Brazilian nationally representative survey of ninth-grade adolescents conducted in 2015 , were used.

Participants: 99791 adolescents (52 015 girls) with a mean age of 14.3 years (range 11-19) participated. All variables were collected through a self-reported questionnaire based on the Global School-Based Student Health Survey. Anxiety-induced sleep disturbance was the outcome. Over $4 \mathrm{~h} / \mathrm{d}$ of TV viewing and daily consumption of ultra-processed foods were the exposures. Body satisfaction, loneliness, self-rated health and eating while watching TV or studying were mediators. Age, ethnicity, food insecurity, type of city (capital or interior), country region and physical activity were covariates. Logistic regression and mediation models (Karlsson-Holm-Breen method) assessed associations.

Results: Both daily ultra-processed food consumption (boys: OR 1·48, $95 \%$ CI 1.30, 1.70; girls: OR 1.46, $95 \%$ CI 1.34, 1.60) and TV viewing (boys: OR $124,95 \%$ CI 1.08, 1.43; girls: OR 1.09, $95 \%$ CI 1.00, 1.19) were associated with higher odds for anxiety-induced sleep disturbance. Loneliness and eating while watching TV or studying (only among girls) consistently mediated the association of both daily ultra-processed food consumption (loneliness: boys $17.4 \%$, girls $23.4 \%$; eat while watching TV or studying: girls $6.8 \%$ ) and TV viewing (loneliness: boys $22.9 \%$, girls $45.8 \%$; eat while watching TV or studying: boys $6.7 \%$, girls $17.9 \%$ ) with anxietyinduced sleep disturbance.

Conclusions: Daily ultra-processed food consumption and TV viewing share mediators and can act in synergic mechanisms in the association with anxiety-induced sleep disturbance. Therefore, future interventions should focus in the reduction of both behaviours in combination.
\end{abstract}

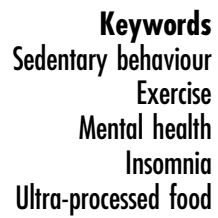

Sleep disturbance and insomnia are highly prevalent among adolescents ${ }^{(1)}$, and it has been suggested that anxiety is a common cause ${ }^{(2)}$. A previous study from the Global School-Based Student Health Survey found that $7.8 \%$ of adolescents had anxiety-induced sleep disturbance, but this prevalence can vary between $1.8 \%$ and $26.8 \%$ depending on the country ${ }^{(3)}$.
Among the risk factors for anxiety-induced sleep disturbance, unhealthy lifestyle behaviours, such as sedentary behaviour, physical inactivity and the consumption of ultra-processed foods, were highlighted ${ }^{(3-6)}$. In addition to their negative independent effects, these behaviours seem to be associated with each other ${ }^{(7-9)}$, a fact that can substantially increase the risk of anxiety-induced sleep 
disturbance. A recent study found that adolescents who reported high daily consumption of ultra-processed foods as well as increased time watching TV had higher prevalence of anxiety-induced sleep disturbance compared to reporting the negative behaviours in isolation ${ }^{(5)}$.

TV viewing and ultra-processed food consumption can share some direct mechanisms in the association with mental disorders during adulthood, such as inflammation, but this relationship is yet to be investigated among adolescents $^{(10,11)}$. Moreover, both ultra-processed foods and TV viewing are associated with body satisfaction ${ }^{(12)}$, loneliness $^{(13)}$ and self-rated general health ${ }^{(14)}$, which have been associated with anxiety-induced sleep disturbance $^{(15-17)}$. Thus, these mechanisms appear to be potential mediators. However, the influence of potential shared mediators of TV viewing and ultra-processed foods in the association with anxiety-induced sleep disturbance is still not clear. Therefore, our aim was to investigate the role of potential shared mediators (i.e. body satisfaction, loneliness, self-rated health and eat while watching TV or studying) in the association of TV viewing and daily ultra-processed food consumption with anxiety-induced sleep disturbance.

\section{Methods}

\section{Sample}

Data from the present study are derived from the Brazilian Adolescent School-Based Health Survey (PeNSE in Portuguese), which is part of the surveillance of risk and protection factors for chronic diseases in Brazil. This survey aims to report on risk factors and health of Brazilian students as well as to identify priority issues for the development of public policies. PeNSE was conducted in $2015^{(18)}$. The survey included a nationally representative sample of students from the final (ninth) year of elementary education, which was selected using a complex multistage, stratified, clustered probability design. The sampling process and methods are detailed elsewhere ${ }^{(18)}$. Briefly, cluster sampling was performed in two stages in capital cities (with schools as primary units and classes as secondary units) and three stages in other municipalities (with municipalities as primary units, schools as secondary units and classes as tertiary units). From 3160 eligible schools, a sample of 3040, with 124227 students, was eligible to be included in the sample. Of these students, 102072 accepted to participate and were at school on the day of interview. All procedures were approved by the National Health Research Ethics Council, and the study was conducted in accordance with the principles expressed in the Declaration of Helsinki (CONEP n. 1.006.467).

\section{Anxiety-induced sleep disturbance}

Anxiety-induced sleep disturbance was assessed with the following question: 'Considering the past 12 months, how frequently do you have insomnia due to worries or concerns? (a) never; (b) rarely; (c) sometimes; (d) frequently; and (e) very frequently', which is based on the question of the Global School-Based Student Health Survey and used in previous studies ${ }^{(3-5)}$. Adolescents reporting frequent sleep disturbance due to worries and concerns were classified as having anxiety-induced sleep disturbance ${ }^{(3-5)}$.

\section{TV viewing}

Participants were asked about their total time watching TV per day, which was also based on the questionnaire of the Global School-Based Student Health Survey. For the analysis, we adopted the cut-point of $>4 \mathrm{~h} / \mathrm{d}$ for TV viewing, which corresponds to high levels of viewing relative to the wider population ${ }^{(6)}$.

\section{Frequency of ultra-processed food consumption}

The consumption of ultra-processed foods was assessed through questionnaires asking about specific ultraprocessed components: industrialized foods (such as hamburger, ham, mortadella, salami, sausage, instant noodles, packet saltiness, salty crackers), fast foods, confectionery (candies, chocolates, chewing gum, lollipops) and soft drinks. The base question was: 'In the last $7 \mathrm{~d}$, on how many days did you eat...?' This was an adaptation based on the NOVA recommendation for ultra-processed food consumption $^{(19)}$. Those who reported daily consumption of at least one group of ultra-processed were classified as 'daily consumers'(8).

\section{Mediators}

Body satisfaction was estimated through the question: 'How do you feel about your body?' Possible answers were: '(a) very satisfied', '(b) satisfied', '(c) indifferent', '(d) dissatisfied' and '(e) very dissatisfied'. Those who indicated options 'a', 'b' or 'c' were considered to be 'satisfied' with their body, and those who reported options ' $d$ ' and 'e' were 'dissatisfied' with their body. Feeling of loneliness was assessed through the question, "When you consider the past 12 months, how often did you feel lonely?' Possible answers were: '(a) never', '(b) rarely', '(c) sometimes', '(d) frequently' and '(e) very frequently'. We considered as at risk those who felt loneliness frequently and very frequently ('yes'), and at no risk if they indicated other options ('no'). Self-rated health was estimated through the question, 'How would you rate your health status?', with the potential answers '(a) very good', '(b) good', '(c) regular', '(d) bad' and '(e) very bad'. We considered them to be at risk if they answered bad or very bad ('poor') and at no risk if they indicated other options ('good'). For the question 'Do you eat when watching TV or studying?', possible answers were: '(a) yes, every day', '(b) yes, 5-6 d a week', '(c) yes, 3-4 d a week', '(d) yes, 1-2 d a week', '(e) rarely' and '(f) no'. We classified the participants as 
having a daily pattern (seven times per week) and no daily pattern if $\leq 6$ times per week.

\section{Covariates}

Chronological age and ethnicity were self-reported by participants; ethnicity was classified as white and nonwhite in our study. Food insecurity was used as a proxy for socioeconomic status. Participants were asked 'During the past $30 \mathrm{~d}$, how often did you go hungry because there was not enough food in your home?' Answer options were classified as 'never', 'rarely/sometimes' and 'most of the time/always'. Physical activity was assessed using questions related to transport, leisure time as well as physical education classes, which has been previously described ${ }^{(20)}$. We used the sum of all domains as our final indicators, with the cut-off point of $300 \mathrm{~min} /$ week, which is a frequently used cut-off point and also corresponds to high levels of physical activity relative to the wider population ${ }^{(20)}$. Moreover, type of city (capital or interior) and country regions (north, northeast, south, southeast, midwest) were also used as a covariate into the analyses.

\section{Statistical procedures}

The characteristics of the sample as well as the comparison between included and excluded participants were described by sex using frequencies and 95\% CI. The isolated association of daily ultra-processed food consumption and TV viewing with anxiety-induced sleep disturbance was assessed through logistic regression models, with a crude model (model 1) and an adjusted model (model 2 adjusted for age group, ethnicity, food insecurity, country region, type of city and physical activity).

After this, mediation models were conducted to assess the influence of potential mediators (body satisfaction, loneliness, self-rated health and eat while watching TV or studying) in the associations of daily ultra-processed food consumption and TV viewing with anxiety-induced sleep disturbance (Fig. 1). The Karlson-Holm-Breen method was used for the mediation ${ }^{(21)}$, creating different models for each mediator. This method was applied in logistic regression models and decomposes the total effect of a variable into direct and indirect effects. This estimation also provides the percentage of explanation by the mediator (mediated percentage). The mediation models were also adjusted for age group, ethnicity, food insecurity,

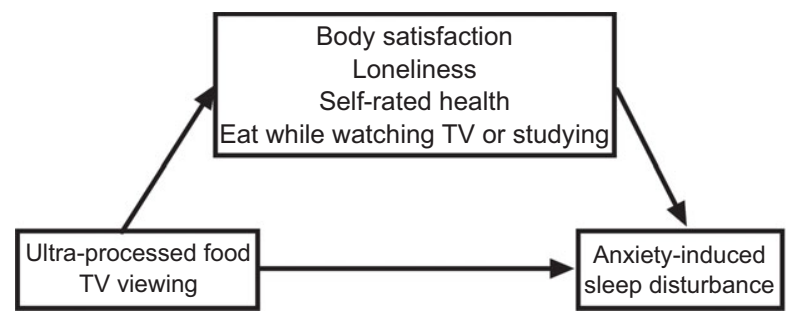

Fig. 1 Theoretical model country region, type of city and physical activity. As an assumption for the use of the Karlson-Holm-Breen method, we tested the interactions between exposures and mediators in the association with the outcome and found no significant interactions. Sampling weights (accounted for the type of school (public or private), number of classes inside each school, valid questionnaires inside each class, number of classes selected in the school and representativeness of all country regions), strata and primary sample unit were accounted in all analyses, conducted in Stata 15.1.

\section{Results}

From the initial sample (102 072 adolescents), 2281 presented missing data in at least one variable of the present study and were excluded. The excluded sample presented a higher proportion of boys (66.6\% v. 48.3\%), adolescents aged $>14$ years $(42 \cdot 2 \% v \cdot 30 \cdot 4 \%)$, but with similar rates of elevated food insecurity (most of the time/always), region and type of city (Table 1$)$. Therefore, the final sample composed of 99791 adolescents (52 038 girls).

Table 2 shows the characteristics of the sample according to sex. Girls had higher prevalence of anxiety-induced

Table 1 Characteristics of sample according to included and excluded sample

\begin{tabular}{|c|c|c|c|c|}
\hline & \multicolumn{2}{|c|}{ Included (n 99 791) } & \multicolumn{2}{|c|}{ Excluded ( $n$ 2281) } \\
\hline & $\begin{array}{l}\text { Relative } \\
\text { frequencies }\end{array}$ & $95 \% \mathrm{Cl}$ & $\begin{array}{l}\text { Relative } \\
\text { frequencies }\end{array}$ & $95 \% \mathrm{Cl}$ \\
\hline \multicolumn{5}{|l|}{ Sex } \\
\hline Boy & $48 \cdot 3$ & $47 \cdot 7,48 \cdot 9$ & $66 \cdot 6$ & $63.1,69.9$ \\
\hline Girl & 51.7 & $51 \cdot 1,52 \cdot 3$ & 33.4 & $30 \cdot 1,36 \cdot 9$ \\
\hline \multicolumn{5}{|l|}{ Age group } \\
\hline $\begin{array}{l}\text { Less than } \\
14 \text { years }\end{array}$ & $18 \cdot 3$ & $17 \cdot 9,18 \cdot 8$ & $13 \cdot 8$ & $11 \cdot 2,16 \cdot 8$ \\
\hline 14 years & $51 \cdot 2$ & $50 \cdot 6,51 \cdot 7$ & $44 \cdot 0$ & $40 \cdot 4,47 \cdot 7$ \\
\hline $\begin{array}{c}\text { More than } \\
14 \text { years }\end{array}$ & $30 \cdot 4$ & $30 \cdot 0,31 \cdot 0$ & $42 \cdot 2$ & $38.7,45 \cdot 9$ \\
\hline \multicolumn{5}{|l|}{ Ethnicity* } \\
\hline White & $36 \cdot 2$ & $35 \cdot 7,36 \cdot 8$ & $32 \cdot 5$ & $29 \cdot 0,36 \cdot 2$ \\
\hline Other & $63 \cdot 8$ & $63 \cdot 2,64 \cdot 3$ & 67.5 & $63 \cdot 8,71 \cdot 0$ \\
\hline \multicolumn{5}{|l|}{ Food insecurity } \\
\hline Never & $78 \cdot 6$ & $78 \cdot 1,79 \cdot 0$ & $79 \cdot 0$ & $75 \cdot 9,81 \cdot 8$ \\
\hline $\begin{array}{l}\text { Rarely/ } \\
\text { sometimes }\end{array}$ & $18 \cdot 9$ & $18 \cdot 5,19 \cdot 4$ & $17 \cdot 2$ & $14 \cdot 7,20 \cdot 0$ \\
\hline $\begin{array}{l}\text { Most of the } \\
\text { time/always }\end{array}$ & $2 \cdot 5$ & $2 \cdot 3,2 \cdot 7$ & 3.9 & $2 \cdot 6,5 \cdot 6$ \\
\hline \multicolumn{5}{|l|}{ Country region } \\
\hline North & $9 \cdot 6$ & $9 \cdot 4,9.8$ & 8.3 & $7 \cdot 2,9 \cdot 5$ \\
\hline Northeast & $27 \cdot 7$ & $27 \cdot 3,28 \cdot 1$ & $27 \cdot 1$ & $24 \cdot 5,29 \cdot 8$ \\
\hline Southeast & $43 \cdot 4$ & $42 \cdot 8,44 \cdot 0$ & 41.7 & $37 \cdot 7,45 \cdot 7$ \\
\hline South & $11 \cdot 8$ & $11 \cdot 5,12 \cdot 1$ & $13 \cdot 5$ & $11.4,15.9$ \\
\hline Midwest & 7.4 & $7 \cdot 3,7 \cdot 6$ & 9.5 & $8 \cdot 3,10 \cdot 8$ \\
\hline \multicolumn{5}{|l|}{ Type of city } \\
\hline Capital & $23 \cdot 0$ & $22 \cdot 6,23 \cdot 3$ & $24 \cdot 8$ & $22 \cdot 3,27 \cdot 5$ \\
\hline Interior & $77 \cdot 0$ & $76 \cdot 7,77 \cdot 4$ & $75 \cdot 2$ & $72 \cdot 5,77 \cdot 7$ \\
\hline
\end{tabular}

Note. Values are presented in absolute frequencies, weighted relative frequencies as well as $95 \% \mathrm{Cl}$.

${ }^{*} n$ of excluded $=2173$.

t $n$ of excluded $=2097$. 
Table 2 Characteristics of sample according to sex

\begin{tabular}{|c|c|c|c|c|c|c|}
\hline & \multirow[b]{2}{*}{$n$} & \multicolumn{2}{|c|}{ Boys ( $n 47776)$} & \multirow[b]{2}{*}{$n$} & \multicolumn{2}{|c|}{ Girls ( $n 52$ 015) } \\
\hline & & Relative frequencies & $95 \% \mathrm{Cl}$ & & Relative frequencies & $95 \% \mathrm{Cl}$ \\
\hline \multicolumn{7}{|l|}{ Age group } \\
\hline Less than 14 years & 6988 & $16 \cdot 1$ & $15 \cdot 5,16 \cdot 8$ & 9977 & $20 \cdot 4$ & $19 \cdot 8,21 \cdot 1$ \\
\hline 14 years & 23103 & $48 \cdot 9$ & $48 \cdot 1,49 \cdot 7$ & 27525 & $53 \cdot 3$ & $52 \cdot 5,54 \cdot 0$ \\
\hline More than 14 years & 17685 & $35 \cdot 0$ & $34 \cdot 3,35 \cdot 7$ & 14513 & $26 \cdot 3$ & $25 \cdot 7,26 \cdot 9$ \\
\hline \multicolumn{7}{|l|}{ Ethnicity } \\
\hline White & 16535 & 37.5 & $36 \cdot 7,38 \cdot 3$ & 16573 & $35 \cdot 0$ & $34 \cdot 2,35 \cdot 8$ \\
\hline Other & 31241 & 62.5 & $61 \cdot 7,63 \cdot 3$ & 35442 & $65 \cdot 0$ & $64 \cdot 2,65 \cdot 7$ \\
\hline \multicolumn{7}{|l|}{ Food insecurity } \\
\hline Never & 37327 & $78 \cdot 8$ & $78 \cdot 1,79 \cdot 4$ & 40357 & $78 \cdot 8$ & $78 \cdot 1,79 \cdot 4$ \\
\hline Rarely/sometimes & 9423 & $19 \cdot 2$ & $18 \cdot 5,19 \cdot 8$ & 10157 & $19 \cdot 2$ & $18.5,19.8$ \\
\hline Most of the time/always & 1026 & $2 \cdot \overline{1}$ & $1 \cdot 8,2 \cdot 3$ & 1501 & $2 \cdot 1$ & $1 \cdot 8,2 \cdot 3$ \\
\hline \multicolumn{7}{|l|}{ Country region } \\
\hline North & 11347 & $9 \cdot 6$ & $9.5,9.6$ & 12048 & 9.6 & $9 \cdot 6,9 \cdot 7$ \\
\hline Northeast & 16452 & $26 \cdot 4$ & $26 \cdot 3,26 \cdot 4$ & 19076 & 29.0 & $28 \cdot 9,29 \cdot 1$ \\
\hline Southeast & 8504 & 44.5 & $44.4,44 \cdot 6$ & 8945 & $42 \cdot 4$ & $42 \cdot 3,42 \cdot 5$ \\
\hline South & 4711 & $12 \cdot 0$ & $12 \cdot 0,12 \cdot 1$ & 4893 & 11.6 & $11 \cdot 6,11 \cdot 7$ \\
\hline Midwest & 6762 & 7.5 & $7 \cdot 5,7.6$ & 7053 & $7 \cdot 3$ & $7 \cdot 3,7 \cdot 4$ \\
\hline \multicolumn{7}{|l|}{ Type of city } \\
\hline Capital & 24130 & $23 \cdot 2$ & $23 \cdot 1,23 \cdot 2$ & 25966 & $22 \cdot 8$ & $22 \cdot 7,22 \cdot 8$ \\
\hline Interior & 23646 & $76 \cdot 8$ & $76 \cdot 8,76 \cdot 9$ & 26049 & $77 \cdot 2$ & $77 \cdot 1,77 \cdot 3$ \\
\hline \multicolumn{7}{|l|}{ Habitual PA } \\
\hline$\leq 299 \mathrm{~min} /$ week & 26750 & $56 \cdot 0$ & $55 \cdot 1,56 \cdot 8$ & 38590 & 74.6 & $73 \cdot 9,75 \cdot 2$ \\
\hline$\geq 300 \mathrm{~min} / \mathrm{week}$ & 21026 & $44 \cdot 0$ & $43 \cdot 2,44 \cdot 9$ & 13425 & $25 \cdot 4$ & $24 \cdot 8,26 \cdot 1$ \\
\hline \multicolumn{7}{|c|}{ Anxiety-induced sleep disturbance } \\
\hline No & 44400 & 93.1 & $92 \cdot 7,93.5$ & 43883 & 84.6 & $84 \cdot 0,85 \cdot 1$ \\
\hline Yes & 3376 & $6 \cdot 8$ & $6 \cdot 4,7 \cdot 3$ & 8132 & $15 \cdot 4$ & $14 \cdot 9,16 \cdot 0$ \\
\hline \multicolumn{7}{|c|}{ Ultra-processed foods consumption } \\
\hline$\leq 6$ times/week & 31063 & 63.7 & $62 \cdot 9,64.5$ & 29799 & $56 \cdot 5$ & $55 \cdot 7,57 \cdot 3$ \\
\hline$\overline{7}$ times/week & 16713 & $36 \cdot 3$ & $35 \cdot 5,37 \cdot 1$ & 22216 & 43.5 & $42 \cdot 7,44 \cdot 3$ \\
\hline \multicolumn{7}{|l|}{ TV-viewing } \\
\hline$\leq 3.99 \mathrm{~h} / \mathrm{d}$ & 33189 & $68 \cdot 3$ & $67 \cdot 5,69 \cdot 0$ & 33931 & $63 \cdot 1$ & $62 \cdot 4,63 \cdot 8$ \\
\hline$\geq 4 \mathrm{~h} / \mathrm{d}$ & 14587 & $31 \cdot 7$ & $31 \cdot 0,32 \cdot 5$ & 18084 & $36 \cdot 9$ & $36 \cdot 2,37 \cdot 6$ \\
\hline \multicolumn{7}{|c|}{ Eat while watching TV or studying } \\
\hline$\leq 6$ times/week & 26902 & $54 \cdot 1$ & $53 \cdot 3,54.9$ & 30635 & $57 \cdot 0$ & $56 \cdot 2,57 \cdot 7$ \\
\hline$\overline{7}$ times/week & 20874 & $45 \cdot 9$ & $45 \cdot 1,46 \cdot 7$ & 21380 & $43 \cdot 0$ & $42 \cdot 2,43 \cdot 8$ \\
\hline \multicolumn{7}{|l|}{ Body image satisfaction } \\
\hline Satisfied & 37105 & 77.9 & $77 \cdot 2,78 \cdot 6$ & 34390 & $68 \cdot 5$ & $67 \cdot 8,69 \cdot 2$ \\
\hline Dissatisfied & 10671 & $22 \cdot 1$ & $21 \cdot 4,22 \cdot 8$ & 17625 & 31.5 & $30 \cdot 8,32 \cdot 2$ \\
\hline \multicolumn{7}{|l|}{ Loneliness } \\
\hline No & 42819 & $89 \cdot 8$ & $89 \cdot 3,90 \cdot 3$ & 40147 & $77 \cdot 7$ & $77 \cdot 0,78 \cdot 3$ \\
\hline Yes & 4957 & $10 \cdot 2$ & $9 \cdot 7,10 \cdot 7$ & 11868 & $22 \cdot 3$ & $21 \cdot 7,23 \cdot 0$ \\
\hline \multicolumn{7}{|l|}{ Self-rated health } \\
\hline Good & 36606 & 77.9 & $77 \cdot 2,78 \cdot 6$ & 34728 & 68.5 & $67 \cdot 8,69 \cdot 2$ \\
\hline Poor & 11170 & $22 \cdot 1$ & $21 \cdot 4,22 \cdot 8$ & 17287 & 31.5 & $30 \cdot 8,32 \cdot 2$ \\
\hline
\end{tabular}

Note. Values are presented in absolute frequencies, weighted relative frequencies as well as $95 \% \mathrm{Cl}$.

sleep disturbance (girls 15.4\%, 95\% CI 14.9\%, 16.0\% $v$. boys: $6.8 \%, 95 \%$ CI $6.4 \%, 7 \cdot 3 \%$ ), daily ultra-processed food consumption (girls: OR $43 \cdot 5,95 \% \mathrm{CI} 42 \cdot 7,44 \cdot 3 v$. boys: OR 36.3, $95 \%$ CI $35 \cdot 5,37 \cdot 1$ ), body dissatisfaction (girls: OR $31 \cdot 5,95 \%$ CI $30 \cdot 8,32 \cdot 2 v$. boys: OR $22 \cdot 1,95 \%$ CI $21 \cdot 4,22 \cdot 8)$, loneliness (girls: OR 22.3, $95 \%$ CI 21.7, $23.0 v$. boys: OR $10 \cdot 2,95 \%$ CI $9 \cdot 7,10 \cdot 7$ ) and poor self-rated health (girls: OR 31.5, $95 \%$ CI 30.8, $32 \cdot 2 v$. boys: OR 22.1, $95 \%$ CI $21 \cdot 4,22 \cdot 8)$.

The isolated associations of daily ultra-processed food consumption and TV viewing with anxiety-induced insomnia are presented in Table 3. Both daily ultra-processed food consumption (boys: OR 1.48, $95 \%$ CI 1.30, 1.70; girls: OR $1 \cdot 46,95 \%$ CI 1.34, 1.60) and TV viewing (boys: OR 1.24,
$95 \%$ CI 1.08, 1.43; girls: OR 1.09, $95 \%$ CI 1.00, 1.19) were associated with higher odds for anxiety-induced sleep disturbance.

Considering mediation models (Table 4), we observed that loneliness and eat while watching TV or studying consistently mediated the association of both daily ultraprocessed food consumption (loneliness: boys $17 \cdot 4 \%$, girls $23.4 \%$; eat while watching TV or studying: girls $6.8 \%$ ) and TV viewing (loneliness: boys $22.9 \%$, girls $45.8 \%$; eat while watching TV or studying: boys $6.7 \%$, girls $17.9 \%$ ) with anxiety-induced sleep disturbance. Self-rated health mediated the association between daily ultra-processed food consumption and anxiety-induced sleep disturbance only among girls (9.5\%). Also, self-rated health (boys 
Table 3 Associations between daily ultra-processed food consumption and TV-viewing with anxiety-induced sleep disturbance among adolescents

\begin{tabular}{lccrr}
\hline & Boys OR & $95 \% \mathrm{Cl}$ & Girls OR & $95 \% \mathrm{Cl}$ \\
\hline Ultra-processed food (Ref: $\leq 6$ times/week) & & & & \\
$\quad$ Model 1 (crude model) & 1.52 & $1.34,1.74$ & 1.49 & $1.37,1.62$ \\
Model 2 (adjusted model) & 1.48 & $1.30,1.70$ & 1.46 & $1.34,1.60$ \\
TV-viewing (Ref: $\leq 3.99 \mathrm{~h} / \mathrm{d}$ ) & & & 1.16 & $1.06,1.27$ \\
Model 1 (crude model) & 1.32 & $1.15,1.51$ & 1.09 & $1.00,1.19$ \\
Model 2 (adjusted model) & 1.24 & $1.08,1.43$ & & \\
\hline
\end{tabular}

$\mathrm{Cl}$, Confidence interval.

Note. Adjusted for age group, ethnicity, food insecurity, country region, type of city (capital or interior) and physical activity.

Table 4 Mediation models for the association of daily ultra-processed food and TV-viewing with anxiety-induced sleep disturbance among adolescents

\begin{tabular}{|c|c|c|c|c|c|c|c|c|c|c|c|}
\hline Mediator & Sex & $\begin{array}{c}\text { Total } \\
\text { effect OR }\end{array}$ & $95 \% \mathrm{Cl}$ & $P$ & $\begin{array}{l}\text { Direct } \\
\text { effect OR }\end{array}$ & $95 \% \mathrm{Cl}$ & $P$ & $\begin{array}{l}\text { Indirect } \\
\text { effect OR }\end{array}$ & $95 \% \mathrm{Cl}$ & $P$ & $\begin{array}{c}\% \\
\text { Mediator }\end{array}$ \\
\hline \multicolumn{12}{|c|}{ Daily ultra-processed food } \\
\hline \multirow[t]{2}{*}{ Body satisfaction } & Boys & 1.49 & $1 \cdot 31,1 \cdot 71$ & $<0.001$ & 1.49 & $1.30,1.70$ & $<0.001$ & 1.00 & $0.99,1.01$ & 0.922 & - \\
\hline & Girls & 1.47 & $1 \cdot 35,1 \cdot 61$ & $<0.001$ & 1.46 & $1.33,1.59$ & $<0.001$ & 1.01 & $1.00,1.02$ & 0.045 & $3 \cdot 0$ \\
\hline \multirow[t]{2}{*}{ Loneliness } & Boys & 1.46 & $1.27,1.68$ & $<0.001$ & 1.37 & $1.19,1.58$ & $<0.001$ & 1.07 & $1.04,1.10$ & $<0.001$ & $17 \cdot 4$ \\
\hline & Girls & 1.48 & $1.35,1.63$ & $<0.001$ & $1 \cdot 35$ & $1.23,1.48$ & $<0.001$ & $1 \cdot 10$ & $1.07,1.12$ & $<0.001$ & 23.4 \\
\hline \multirow[t]{2}{*}{ Self-rated health } & Boys & 1.49 & $1 \cdot 30,1 \cdot 70$ & $<0.001$ & 1.47 & $1 \cdot 29,1 \cdot 68$ & $<0.001$ & 1.01 & $1.00,1.02$ & 0.054 & - \\
\hline & Girls & 1.47 & $1.35,1.61$ & $<0.001$ & 1.42 & $1.30,1.55$ & $<0.001$ & 1.04 & $1.03,1.05$ & $<0.001$ & 9.5 \\
\hline \multirow{2}{*}{$\begin{array}{l}\text { Eat while watching } \\
\text { TV or studying }\end{array}$} & Boys & 1.49 & $1 \cdot 30,1 \cdot 70$ & $<0.001$ & 1.46 & $1.28,1.67$ & $<0.001$ & 1.02 & $1.00,1.04$ & 0.062 & - \\
\hline & Girls & 1.47 & $1.34,1.60$ & $<0.001$ & 1.43 & $1.31,1.56$ & $<0.001$ & 1.03 & $1.01,1.04$ & $<0.001$ & $6 \cdot 8$ \\
\hline \multicolumn{12}{|l|}{ TV-viewing } \\
\hline \multirow[t]{2}{*}{ Body satisfaction } & Boys & 1.24 & $1.08,1.43$ & 0.002 & 1.22 & $1.06,1.40$ & 0.005 & 1.02 & $1.01,1.03$ & 0.007 & $9 \cdot 0$ \\
\hline & Girls & 1.09 & $1.00,1.19$ & 0.062 & 1.07 & $0.98,1.17$ & 0.151 & 1.02 & $1.01,1.03$ & 0.001 & $22 \cdot 9$ \\
\hline \multirow[t]{2}{*}{ Loneliness } & Boys & $1 \cdot 22$ & $1.06,1.42$ & 0.007 & $1 \cdot 17$ & $1.01,1.35$ & 0.037 & 1.05 & $1.02,1.07$ & $<0.001$ & $22 \cdot 9$ \\
\hline & Girls & 1.09 & $0.99,1.20$ & 0.087 & 1.05 & $0.95,1.15$ & 0.354 & 1.04 & $1.01,1.06$ & 0.002 & $45 \cdot 8$ \\
\hline \multirow[t]{2}{*}{ Self-rated health } & Boys & $1 \cdot 24$ & $1.08,1.43$ & 0.002 & 1.20 & $1.05,1.38$ & 0.008 & 1.03 & $1.02,1.05$ & $<0.001$ & $14 \cdot 2$ \\
\hline & Girls & 1.09 & $0.99,1.19$ & 0.066 & 1.07 & $0.97,1.17$ & 0.170 & 1.02 & $1.01,1.03$ & $<0.001$ & $25 \cdot 3$ \\
\hline \multirow{2}{*}{$\begin{array}{l}\text { Eat while watching } \\
\text { TV or studying }\end{array}$} & Boys & $1 \cdot 24$ & $1.08,1.43$ & 0.002 & $1 \cdot 23$ & $1.07,1.41$ & 0.004 & 1.01 & $1.01,1.03$ & 0.017 & $6 \cdot 7$ \\
\hline & Girls & 1.09 & $0.99,1.19$ & 0.067 & 1.07 & $0.98,1.17$ & 0.133 & 1.02 & $1.00,1.03$ & 0.004 & 17.9 \\
\hline
\end{tabular}

OR, odds ratio; $\mathrm{Cl}$, confidence interval.

Note. Adjusted by age group, ethnicity, food insecurity, country region, type of city (capital or interior) and physical activity.

$14.2 \%$, girls $25.3 \%$ ) and body satisfaction (boys $9 \cdot 0 \%$, girls $22.9 \%$ ) mediated the association between TV viewing and anxiety-induced sleep disturbance in both sexes.

\section{Discussion}

This cross-sectional study aimed to investigate the role of potential shared mediators of the associations of daily ultra-processed food consumption and TV viewing with anxiety-induced sleep disturbance. Our main finding was that daily ultra-processed food consumption and TV viewing have common mediators as loneliness and eat while watching TV or studying, and that the influence of the mediators can vary according to sex. This study advances the investigation of how ultra-processed food consumption and TV viewing, as well as their co-occurrence, can be associated with anxiety-induced sleep disturbance ${ }^{(5)}$. Also, girls self-reported poorer health indicators, including anxiety-induced sleep disturbance, self-rated health, loneliness, body satisfaction as well as higher TV viewing and ultra-processed food consumption.

The present study is consistent with previous findings concerning the independent associations of daily ultraprocessed food consumption and TV viewing with anxiety-induced sleep disturbance ${ }^{(3-6)}$. These independent associations can present different direct mechanisms, such as the blue light emission by television, which is associated with poorer sleep quality ${ }^{(22,23)}$. In addition, there are biological mechanisms linking sitting time and anxiety and sleep disturbance, such as the increase of inflammatory markers like IL-6 and C-reactive protein ${ }^{(10)}$. Similarly, the daily consumption of ultraprocessed foods can present direct roles in the association with anxiety-induced sleep disturbance, that is, the increased consumption of fat, sugar and additives can also be associated with inflammatory markers and consequently sleep disturbance and insomnia ${ }^{(11,24)}$. 
However, these mechanisms have been less studied among adolescents.

However, we investigated the role of psychological/ behavioural mediators such as body satisfaction, loneliness, self-rated health and eat while watching TV or studying. Body satisfaction is strongly related to weight status $^{(25)}$, which can be associated with anxiety ${ }^{(26)}$. However, we only found consistent mediation considering TV viewing, and not daily ultra-processed food consumption. A possible explanation could be through the stigmatization produced by the TV programmes ${ }^{(27,28)}$. Therefore, weight stigmatization can be associated with poorer body satisfaction and consequently higher sleep disturbance ${ }^{(17)}$. Self-rated health was also an important mediator concerning the association between TV viewing and anxiety-induced sleep disturbance. TV viewing is associated with self-rated health ${ }^{(14)}$, and selfrated health is closely associated with sleep disturbance ${ }^{(16)}$.

We found that loneliness has a pivotal role in linking both daily ultra-processed food consumption and TV viewing with anxiety-induced sleep disturbance. This indicates that both behaviours are associated with social exclusion and frequently practised alone. Moreover, eating while watching TV is associated with considerably higher consumption of daily ultra-processed food ${ }^{(9)}$. Therefore, it is also possible to interpret eating while watching TV or studying as an indicator of both behaviours co-occurring ${ }^{(7,8)}$. Thus, this co-occurrence can be a risk factor and links the social isolation caused by TV viewing with the daily consumption of ultra-processed foods, such as snacking and soft drinks consumption ${ }^{(5,7,29)}$, and consequently with anxiety-induced sleep disturbance ${ }^{(30)}$.

We highlight that the associations of TV viewing and ultra-processed food consumption with anxiety-induced sleep disturbance can be bidirectional. Therefore, it is also possible that higher anxiety-induced sleep disturbance can lead to mediators and also to higher TV viewing and consumption of ultra-processed foods ${ }^{(31-33)}$. In this sense, higher sleep disturbance should lead to a lack of energy and consequently higher TV viewing, which could also pass through the mediators such as loneliness and eat while watching TV or studying ${ }^{(32)}$. Also, previous studies have found that sleep deprivation can be associated with different food choices, especially increasing the consumption of sugary foods ${ }^{(31,33)}$

Our findings should be interpreted in the light of potential limitations. The cross-sectional design limits any inference of causality. Mediation models are causal models, and their interpretation is often limited in cross-sectional studies $^{(34)}$. However, there is a clear plausibility for the causal association between both exposures and mediators as well as between mediators and mental health ${ }^{(12-17)}$. Also, there were no interactions between exposures and mediators, indicating that the mediators did not change the association between exposures and outcome, but reduced the degree of association. The consumption of ultraprocessed foods relied on a 7-d recall on days per week, without further information on daily servings and specific calorie contribution of each food, which does not allow the estimation of a specific ultra-processed food's contribution to daily energy demand. It being based on self-report, this information is prone to recall bias ( $7-\mathrm{d}$ recall). Also, we did not consider potential unmeasured confounders such as weight status, pubertal status, parental history of mental disorders and peer influences on diet and TV viewing because such information was not available. On the other hand, we included a large nationally representative sample of Brazilian adolescents. To our knowledge, this is the first study aiming to investigate the potential common mediators of the associations of daily ultra-processed food consumption and TV viewing with anxiety-induced sleep disturbance. In terms of practical applications, these results not only reinforce the need of public health policies to reduce sedentary behaviours and unhealthy eating habits among adolescents but also indicate ways to build effective actions, focusing on the shared mediators that link TV viewing and ultra-processed food consumption with anxiety-induced sleep disturbance.

We conclude that daily ultra-processed food consumption and TV viewing share mediators in the association with anxiety-induced sleep disturbance, such as loneliness and eating while watching TV or studying. Therefore, both unhealthy lifestyle behaviours can act in synergic mechanisms in the association with anxiety disorders.

\section{Acknowledgements}

Acknowledgements: We gratefully acknowledge the contributions of all participants of the present research as well as the IBGE staff for data collection. Financial support: This research received no specific grant from any funding agency in the public, commercial or not-for-profit sectors. A.W. is supported by the São Paulo Research Foundation (FAPESP) with a PhD scholarship (FAPESP process 2019/ 24124-7). E.H. is supported by a National Health and Medical Research Council Early Career Fellowship (APP1156909). This paper presents independent research. The views expressed in this publication are those of the authors and not necessarily those of the acknowledged institutions. Conflict of interest: There are no conflicts of interest. Authorship: All authors listed met the conditions required for full authorship. Concept and study design, data analysis: A.O.W. and D.R.S. Interpretation of data: A.O.W., E.H. and D.R.S. Drafting of manuscript: A.O.W. Critical revision and approval of the manuscript for important intellectual content: all authors. Ethics of human subject participation: All procedures performed in the original studies involving human participants were approved by the National Health Research Ethics Council (CONEP: 1.006.467/2015) in accordance with the ethical standards of the institutional and/or national research committee 
and with the 1964 Helsinki Declaration and its later amendments or comparable ethical standards. Data sharing statement: All data are open published as microdata at https://www.ibge.gov.br/estatisticas/sociais/populacao/ 9134-pesquisa-nacional-de-saude-do-escolar.html?=\&t= microdados.

\section{References}

1. Zambotti M, Goldstone A, Colrain IM et al. (2018) Insomnia disorder in adolescence: diagnosis, impact, and treatment. Sleep Med Rev 39, 12-24.

2. Mullin BC \& Simon SL (2017) Managing insomnia symptoms among adolescents with anxiety disorders. Evid Based Pract Child Adolesc Ment Health 2, 123-138.

3. Vancampfort D, Van Damme T, Stubbs B et al. (2019) Sedentary behavior and anxiety-induced sleep disturbance among 181,093 adolescents from 67 countries: a global perspective. Sleep Med 58, 19-26.

4. Khan A \& Uddin R (2020) Is consumption of fast-food and carbonated soft drink associated with anxiety-induced sleep disturbance among adolescents? A population-based study. Clin Nutr ESPEN 36, 162-165.

5. Werneck AO, Vancampfort D, Oyeyemi AL et al. (2020) Joint association of ultra-processed food and sedentary behavior with anxiety-induced sleep disturbance among Brazilian adolescents. J Affect Disord 266, 135-142.

6. Werneck AO, Vancampfort D, Oyeyemi AL et al. (2018) Associations between TV viewing, sitting time, physical activity and insomnia among 100,839 Brazilian adolescents. Psychiatr Res 269, 700-706.

7. Ashdown-Franks G, Vancampfort D, Firth J et al. (2019) Association of leisure-time sedentary behavior with fast food and carbonated soft drink consumption among 133,555 adolescents aged 12-15 years in 44 low- and middle-income countries. Int J Behav Nutr Phys Act 16, 35.

8. Costa C dos S, Flores TR, Wendt A et al. (2018) Sedentary behavior and consumption of ultra-processed foods by Brazilian adolescents: Brazilian National School Health Survey (PeNSE), 2015. Cad Saúde Pública 34, e00021017.

9. Martines RM, Machado PP, Neri DA et al. (2019) Association between watching TV whilst eating and children's consumption of ultraprocessed foods in United Kingdom. Matern Child Nutr 15, e12819.

10. Endrighi R, Steptoe A \& Hamer M (2016) The effect of experimentally induced sedentariness on mood and psychobiological responses to mental stress. Br J Psychiatr 208, 245-251.

11. Firth J, Veronese N, Cotter J et al. (2019) What is the role of dietary inflammation in severe mental illness? A review of observational and experimental findings. Front Psychiatr 10, 350 .

12. Matias TS, Lopes MVV, de Mello GT et al. (2019) Clustering of obesogenic behaviors and association with body image among Brazilian adolescents in the national school-based health survey (PeNSE 2015). Prev Med Rep 16, 101000.

13. Ferrer-Cascales R, Albaladejo-Blázquez N, Ruiz-Robledillo N et al. (2018) Low adherence to the Mediterranean diet in isolated adolescents: the mediation effects of stress. Nutrients 10, 1894.

14. Osera T, Awai M, Kobayashi M et al. (2017) Relationship between self-rated health and lifestyle and food habits in Japanese high school students. Behav Sci 7, 71 .
15. Ben Simon E \& Walker MP (2018) Sleep loss causes social withdrawal and loneliness. Nat Commun 9, 3146.

16. Conklin AI, Yao CA \& Richardson CG (2019) Chronic sleep disturbance, not chronic sleep deprivation, is associated with self-rated health in adolescents. Prev Med 124, $11-16$.

17. Kelly Y, Zilanawala A, Booker C et al. (2018) Social media use and adolescent mental health: findings from the UK millennium cohort study. EClinicalMedicine 6, 59-68.

18. Instituto Brasileiro de Geografia e Estatística - IBGE (2016) Brazilian School-Based Health Survey - PeNSE. Brasília, Brazil: IBGE.

19. Monteiro CA, Cannon G, Lawrence M et al. (2019) Ultra-Processed Foods, Diet Quality, and Health Using the NOVA Classification System. Rome: Food and Agriculture Organization of the United Nations - FAO.

20. Werneck AO, Oyeyemi AL, Fernandes RA et al. (2018) Regional socioeconomic inequalities in physical activity and sedentary behavior among Brazilian adolescents. J Phys Act Health 15, 338-344.

21. Breen R, Karlson KB \& Holm A (2013) Total, direct, and indirect effects in Logit and Probit models. Social Method Res 42, 164-191.

22. LeBourgeois MK, Hale L, Chang A-M et al. (2017) Digital media and sleep in childhood and adolescence. Pediatrics 140, S92-S96.

23. Tähkämö L, Partonen T \& Pesonen A-K (2019) Systematic review of light exposure impact on human circadian rhythm. Chronobiol Int 36, 151-170.

24. Lopes AE da SC, Araújo LF, Levy RB et al. (2019) Association between consumption of ultra-processed foods and serum C-reactive protein levels: cross-sectional results from the ELSA-Brasil study. Sao Paulo Med J 137, 169-176.

25. Reel J, Voelker D \& Greenleaf C (2015) Weight status and body image perceptions in adolescents: current perspectives. Adolesc Health Med Ther 6, 149-158.

26. Vannucci A \& Ohannessian CM (2018) Body image dissatisfaction and anxiety trajectories during adolescence. $J$ Clin Child Adolesc Psychol 47, 785-795.

27. Ata RN \& Thompson JK (2010) Weight bias in the media: a review of recent Research. Obes Facts 3, 41-46.

28. Eisenberg ME, Carlson-McGuire A, Gollust SE et al. (2015) A content analysis of weight stigmatization in popular television programming for adolescents: content analysis of weight stigmatization. Int J Eat Disord $\mathbf{4 8}$, 759-766.

29. Boulos R, Vikre EK, Oppenheimer S et al. (2012) ObesiTV: how television is influencing the obesity epidemic. Physiol Behav 107, 146-153.

30. Eccles AM, Qualter P, Madsen KR et al. (2020) Loneliness in the lives of Danish adolescents: associations with health and sleep. Scand J Public Health 48, 877-887.

31. Beebe DW, Simon S, Summer S et al. (2013) Dietary intake following experimentally restricted sleep in adolescents. Sleep 36, 827-834.

32. Chaput J-P \& Dutil C (2016) Lack of sleep as a contributor to obesity in adolescents: impacts on eating and activity behaviors. Int J Behav Nutr Phys Act 13, 103.

33. Kruger AK, Reither EN, Peppard PE et al. (2014) Do sleep-deprived adolescents make less-healthy food choices? Br J Nutr 111, 1898-1904.

34. Fairchild AJ \& McDaniel HL (2017) Best (but oft-forgotten) practices: mediation analysis. Am J Clin Nutr 105, 1259-1271. 